\title{
Lower Extremity Peripheral Arterial Disease Is an Independent Predictor of Coronary Heart Disease and Stroke Risks in Patients with Type 2 Diabetes Mellitus in China
}

\author{
Xiao-Hong Pang, Jue Han, ${ }^{1}$ Wan-Lan Ye, ${ }^{1}$ Xue Sun, ${ }^{1}$ Yue Ding, ${ }^{1}$ Wen-Juan Huang, \\ Yi-Ming Zhao, ${ }^{1}$ Han-Yu Lou, ${ }^{1}$ Li-Zhen Shan, ${ }^{1}$ Ying-Xiu Kang, ${ }^{1}$ Xiao-Xiao Song, \\ Song-Zhao Zhang, ${ }^{2}$ Wei Gu, ${ }^{1}$ and Peng-Fei Shan ${ }^{1}$ \\ ${ }^{1}$ Department of Endocrinology and Metabolism, The Second Affiliated Hospital Zhejiang University College of Medicine, 88 Jiefang \\ Rd, Hangzhou, Zhejiang 310009, China \\ ${ }^{2}$ Department of Clinical Laboratory, The Second Affiliated Hospital Zhejiang University College of Medicine, 88 Jiefang Rd, \\ Hangzhou, Zhejiang 310009, China
}

Correspondence should be addressed to Peng-Fei Shan; shanpf95@hotmail.com

Received 5 February 2017; Revised 4 April 2017; Accepted 9 April 2017; Published 21 May 2017

Academic Editor: Matteo Monami

Copyright (c) 2017 Xiao-Hong Pang et al. This is an open access article distributed under the Creative Commons Attribution License, which permits unrestricted use, distribution, and reproduction in any medium, provided the original work is properly cited.

\begin{abstract}
We aimed to determine the relationship between lower extremity peripheral arterial disease (PAD), 10-year coronary heart disease (CHD), and stroke risks in patients with type 2 diabetes (T2DM) using the UKPDS risk engine. We enrolled 1178 hospitalized T2DM patients. The patients were divided into a lower extremity PAD group (ankle-brachial index $\leq 0.9$ or $>1.4 ; 88$ patients, 7.5\%) and a non-PAD group (ankle-brachial index $>0.9$ and $\leq 1.4 ; 1090$ patients, 92.5\%). Age; duration of diabetes; systolic blood pressure; the hypertension rate; the use of hypertension drugs, ACEI /ARB, and statins; CHD risk; fatal CHD risk; stroke risk; and fatal stroke risk were significantly higher in the PAD group than in the non-PAD group $(P<0.05$ for all). Logistic stepwise regression analysis indicated that $\mathrm{ABI}$ was an independent predictor of 10-year CHD and stroke risks in T2DM patients. Compared with those in the T2DM non-PAD group, the odds ratios (ORs) for CHD and stroke risk were 3.6 (95\% confidence interval (CI), 2.2-6.0; $P<0.001$ ) and 6.9 (95\% CI, 4.0-11.8; $P<0.001)$ in those with lower extremity PAD, respectively. In conclusion, lower extremity PAD increased coronary heart disease and stroke risks in T2DM.
\end{abstract}

\section{Introduction}

Diabetes patients with peripheral arterial disease (PAD) are at an increased risk for cardiovascular disease $[1,2]$. Lower extremity peripheral arterial disease (PAD) is a common type of PAD in patients with type 2 diabetes mellitus (T2DM). In diabetic patients, PAD can be noninvasively and objectively diagnosed by using the anklebrachial index $(\mathrm{ABI})$; this index can also indicate arterial atherosclerosis at other sites $[3,4]$. A low ABI is related to many known cardiovascular risk factors, including hypertension, diabetes, smoking, dyslipidemia, obesity, and increased serum levels of C-reactive protein [5-7]. A few population-based cohort studies have confirmed that a decrease in the $\mathrm{ABI}$ is highly correlated with an increase in the prevalence rate of coronary artery disease and cerebrovascular disease [8-11].

Currently, a number of methods are available for predicting the 10-year risk of cardiovascular disease in individual subjects, such as the Framingham Risk Score (FRS), the 2013 American College of Cardiology (ACC)/American Heart Association (AHA) risk assessment, and the United Kingdom Prospective Diabetes Study (UKPDS) risk engine. The FRS was derived from the Framingham Heart Study to assess the cardiovascular risk based on age, low-density lipoprotein cholesterol (LDL-c), high-density lipoprotein 
cholesterol (HDL-c), smoking, hypertension, and other factors [12]. Since the FRS was generated using data from the general population, its usefulness in predicting cardiovascular risk in diabetic patients is somewhat limited [13]. The 2013 ACC/AHA risk assessment applies to non-Hispanic American men aged 40-79 years [14]. The UKPDS risk engine, on the other hand, is the commonly used method for the prediction of cardiovascular and cerebrovascular disease risk in T2DM patients. This diabetes-specific risk assessment tool is based on the absolute risk of cardiovascular and cerebrovascular diseases in 5102 patients with newly diagnosed T2DM who were followed up for an average of 10.4 years [15]. Unlike the FRS, the UKPDS risk engine takes into consideration the duration of diabetes and the level of glycosylated hemoglobin (HbAlc).

Some reports have indicated that the ABI abnormality was linked to cardiovascular events, cerebrovascular events, and risk factors in patients with diabetes or metabolic syndrome $[1,9,16,17]$. A study from Hong Kong found that in diabetic patients with a slightly decreased ABI (0.91-0.99), the ABI was associated with increased microvascular and macrovascular complications [18]. Mainland China has the largest population of diabetes patients in the world; however, few studies have investigated the relationship of the ABI with the 10-year coronary heart disease (CHD) and stroke risks in T2DM patients in Mainland China. In this study, we aimed to characterize the above relationship in T2DM patients in China by using the UKPDS risk engine.

\section{Materials and Methods}

2.1. Subjects. This study involved T2DM patients who were admitted to the Department of Endocrinology of the Second Affiliated Hospital Zhejiang University Medical College between April 2008 and April 2013. All participants had been diagnosed with diabetes according to the 1999 World Health Organization diagnostic criteria for the diagnosis and classification of diabetes. In our study, we involved only type 2 diabetes mellitus patients. Those with gestational diabetes, other types of diabetes mellitus, type 1 diabetes mellitus, and GAD antibody positivity were excluded. Further, patients with $\mathrm{CHD}$ and stroke were additionally excluded, leaving a total of 1178 cases that were included in the statistical analysis. This study was approved by the Ethics Committee of the Second Affiliated Hospital Zhejiang University School of Medicine, and all subjects gave informed consent for participation.

2.2. Clinical Indices. A detailed medical history was obtained from each patient, including the patient's age, age at diagnosis of diabetes, smoking history, hypertension, and antihypertensive therapy. Each subject also underwent a detailed physical examination, including height, weight, blood pressure, and body mass index (BMI) measurements. Prior to the blood pressure measurements, the patients were asked to sit for $5 \mathrm{~min}$. Subsequently, two consecutive blood pressure measurements were taken with an electronic blood pressure meter (Kenz BPM SP-1, Japan), and the mean of the two values was used.

2.3. Biochemical Indices. Venous blood was collected in the morning (6:00-9:00 AM) after the patient had fasted for 8-12 hours. The fasting blood glucose, total cholesterol (TC), triglyceride (TG), LDL-c, and HDL-c levels were measured by an Olympus AU4500 automatic chemistry analyzer (Olympus Corporation, Tokyo, Japan). The level of HbA1c was determined by a TOSOH HLC-723G8 automatic glycohemoglobin analyzer (Tosoh Corporation, Yamaguchi 746-0042, Japan).

2.4. ABI Measurement. The ABI was measured by a technician who was blinded to the patient history and biochemical indices. The ABI was determined using Doppler ultrasound and a portable optical volume detector (Vista AVS, Summit Doppler, USA). The patients were asked to take off their shoes and lie in a supine position for $5 \mathrm{~min}$. The upper arm and ankle systolic pressures were measured by slowly moving the ultrasonic probe along the arterial contorts until the strongest information was gotten. The ABI was calculated as the ratio of the ankle systolic blood pressure to the brachial arterial systolic pressure. Blood pressure was measured in both lower extremities and used to calculate the ABI. The lower of the two ABI values thus obtained was used in the subsequent analyses, unless one of the $\mathrm{ABI}$ values was greater than 1.4. The patients were divided into two groups based on the $\mathrm{ABI}$ value as follows: patients with an $\mathrm{ABI} \leq 0.9$ or $\mathrm{ABI}>1.4$ were assigned to the PAD group and those with an $\mathrm{ABI}>0.9$ and $\leq 1.4$ formed the non-PAD group [3].

2.5. UKPDS Risk Engine. The risks of $\mathrm{CHD}$, fatal $\mathrm{CHD}$, stroke, and fatal stroke were calculated by the UKPDS risk engine according to the patient's sex, age at diagnosis of diabetes, smoking, systolic blood pressure, hemoglobin, TC, HDL-c, duration of diabetes, atrial fibrillation, and race [12].

2.6. Statistical Analysis. The SPSS 20 statistical software was used for data analysis. Data were expressed as mean \pm standard deviation or mean (95\% confidence interval). Categorical variables were presented as frequencies, with percentages given in parentheses. The $\mathrm{CHD}$ and stroke risks were assessed after stratifying patients by PAD status and age. We used the Mann-Whitney test or independent $t$-test to compare continuous variables among groups and the chi-square test to compare proportional data. Categorical parameters and risk estimation were evaluated using the chi-square test. Binary logistic regression analysis was used to analyze correlations between categorical variables and risk factors, and multivariate linear regression analysis was used for continuous variables. All statistical tests were two-tailed, and $P<0.05$ was considered significant.

\section{Results}

3.1. Comparison of General Characteristics between Diabetic Patients with PAD and with Non-PAD. Of the 1178 T2DM patients included in this study, 621 were men and 557 were 
TABLE 1: General characteristics of subjects.

\begin{tabular}{|c|c|c|}
\hline & Non-PAD group & PAD group \\
\hline Number & 1090 & 88 \\
\hline Age (years) & $57.2 \pm 12.3$ & $69.8 \pm 11.8^{\#}$ \\
\hline Gender (men/women) & $586 / 504$ & $35 / 53^{*}$ \\
\hline YSDD (years) & $7.3 \pm 6.4$ & $11.0 \pm 7.8^{\#}$ \\
\hline $\mathrm{WC}(\mathrm{cm})$ & $87.9 \pm 10.1$ & $88.0 \pm 10.2$ \\
\hline BMI $\left(\mathrm{kg} / \mathrm{m}^{2}\right)$ & $24.0 \pm 3.6$ & $23.3 \pm 3.2$ \\
\hline SBP (mmHg) & $135.6 \pm 19.5$ & $145.4 \pm 20.3^{\#}$ \\
\hline DBP $(\mathrm{mmHg})$ & $81.8 \pm 11.1$ & $79.5 \pm 12.8$ \\
\hline HbAlc (\%) & $9.6 \pm 2.4$ & $9.5 \pm 2.4$ \\
\hline FBS $(\mathrm{mmol} / \mathrm{L})$ & $9.2 \pm 3.7$ & $8.8 \pm 4.1$ \\
\hline Total cholesterol $(\mathrm{mmol} / \mathrm{L})$ & $4.6(4.5,4.7)$ & $4.5(4.2,4.8)$ \\
\hline Triglycerides $(\mathrm{mmol} / \mathrm{L})$ & $1.9(1.9,2.0)$ & $1.9(1.6,2.2)$ \\
\hline HDL-c $(\mathrm{mmol} / \mathrm{L})$ & $1.2(1.2,1.3)$ & $1.2(1.1,1.3)$ \\
\hline $\mathrm{LDL}-\mathrm{c}(\mathrm{mmol} / \mathrm{L})$ & $2.9(2.9,3.0)$ & $2.9(2.7,3.2)$ \\
\hline Hypertension $(n, \%)$ & $(514,47.2 \%)$ & $(65,73.9 \%)^{\#}$ \\
\hline Smoker $(n, \%)$ & $(368,33.8 \%)$ & $(26,29.5 \%)$ \\
\hline Nonantidiabetic drugs $(n, \%)$ & $(184,16.9 \%)$ & $(6,6.8 \%)^{*}$ \\
\hline Only OAD ( $n, \%)$ & $(471,43.2 \%)$ & $(41,46.6 \%)$ \\
\hline Insulin + OAD $(n, \%)$ & $(435,39.9 \%)$ & $(41,46.6 \%)$ \\
\hline Hypertension drugs $(n, \%)$ & $(456,41.9 \%)$ & $(63,71.6 \%)^{\#}$ \\
\hline $\mathrm{ARB} / \mathrm{ACEI}(n, \%)$ & $(227,20.8 \%)$ & $(38,43.2 \%)^{\#}$ \\
\hline Lipid-lowering drugs $(n, \%)$ & $(157,14.4 \%)$ & $(18,20.5 \%)$ \\
\hline Statins $(n, \%)$ & $(135,12.4 \%)$ & $(18,20.5 \%)^{*}$ \\
\hline Fibrates $(n, \%)$ & $(20,1.8 \%)$ & $(0,0 \%)$ \\
\hline
\end{tabular}

${ }^{*} P<0.05$ and ${ }^{\#} P<0.001$ compared with the non-PAD group. Values are presented as the mean \pm standard deviation; abnormal distribution values are shown as mean $(95 \% \mathrm{CI})$.WC: waist circumference; YSDD: years since diagnosis of diabetes; BMI: body mass index (weight in kilograms/square of the height in meters); SBP: systolic blood pressure; DBP: diastolic blood pressure; FBS: fasting blood glucose; HDL-c: high-density lipoprotein cholesterol; LDL-c: low-density lipoprotein cholesterol; PAD: peripheral arterial disease; $\mathrm{ABI}$ : ankle-brachial index; $\mathrm{OAD}$ : oral antidiabetic drug; ARB: angiotensin receptor blocker; ACEI: angiotensin-converting enzyme inhibitors. $\mathrm{PAD}$ group: $\mathrm{ABI} \leq 0.9$ or $\mathrm{ABI}>1.4$; non-PAD group: $0.9<\mathrm{ABI} \leq 1.4$.

women. Their average age was $58.1 \pm 12.7$ years (range, $21-$ 90 years), and the mean duration of diabetes was $7.6 \pm 6.6$ years (range, 0-36 years). In total, 88 (7.5\%) patients were assigned to the PAD group, and 1090 (92.5\%) patients were included in the non-PAD group based on their ABI values. Among the 88 patients in the PAD group, 81 (6.9\%) had an $\mathrm{ABI} \leq 0.9$ and $7(0.6 \%)$ had an $\mathrm{ABI}>1.4$. Age; duration of diabetes; systolic blood pressure; hypertension rate; and the use of hypertension drugs, ACEI (angiotensin-converting enzyme inhibitor)/ARB (angiotensin receptor blocker), and statins were significantly higher in the PAD group than in the non-PAD group $(P<0.05$ for all; Table 1$)$. Sex distribution significantly differed between the two groups, with female patients being much more likely to have an abnormal $\mathrm{ABI}$ and therefore be included in the PAD group $(P<0.05)$.

3.2. Relationship of PAD with CHD and Stroke Risks. CHD risk, fatal $\mathrm{CHD}$ risk, stroke risk, and fatal stroke risk were
TABLE 2: Comparison of CHD and stroke risks.

\begin{tabular}{lcc}
\hline & Non-PAD group & PAD group \\
\hline$N$ & 1090 & 88 \\
CHD risk (\%) & $20.5(19.6-21.4)$ & $35.1(30.7-39.5)^{\#}$ \\
Fatal CHD risk (\%) & $15.1(14.3-16.0)$ & $29.7(25.6-33.8)^{\#}$ \\
Stroke risk (\%) & $9.3(8.6-10.0)$ & $26.3(21.7-30.9)^{\#}$ \\
Fatal stroke risk (\%) & $1.5(1.3-1.6)$ & $4.4(3.5-5.4)^{\#}$ \\
\hline
\end{tabular}

${ }^{\#} P<0.001$ compared with the non-PAD group. Values are expressed as mean $(95 \% \mathrm{CI})$. PAD was defined as an $\mathrm{ABI} \leq 0.9$ or $>1.4$. CHD: coronary heart disease; PAD: peripheral arterial disease; $\mathrm{ABI}$ : ankle-brachial index.

significantly higher in the PAD group than in the non-PAD group $(P<0.05$ for all; Table 2$)$. Spearman correlation analysis indicated that the ABI was negatively correlated with age $(r=-0.144, P<0.01)$, CHD risk $(r=-0.066, P<0.01)$, stroke risk $(r=-0.116, P<0.01)$, and diabetes duration $(r=-0.069, P<0.05)$, while it was positively correlated with diastolic blood pressure $(r=0.078, P<0.01)$ and BMI $(r=0.075, P<0.05)$. The ABI was not correlated with HbA1c, systolic blood pressure, TC, TG, HDL, and LDL.

Considering that age is the most important factor affecting the $\mathrm{ABI}$ and $\mathrm{CHD}$ and stroke risks [19], we stratified the patients by age, in groups of 10 years, and calculated the UKPDS risk scores in both study groups (Figure 1). The results revealed that $\mathrm{CHD}$ and stroke risks gradually increased with age in both the PAD and non-PAD groups. Furthermore, the CHD risk, fatal CHD risk, stroke risk, and fatal stroke risk were higher in the PAD group than in the non-PAD for each age group.

3.3. Effect of PAD on the UKPDS Risk. The UKPDS CHD risk, fatal CHD risk, stroke risk, and fatal stroke risk were used as the dependent variables, and age, diabetes duration, PAD, HbA1c, TC, TG, HDL, LDL, BMI, systolic blood pressure, diastolic blood pressure, smoking, and sex were used as independent variables in a linear regression analysis. The results showed that age, diabetes duration, PAD, and sex were included in the linear regression equation (Table 3 ). We then performed a binary logistic regression analysis with the following dependent variables: age $>50$ years, PAD, elevated $\mathrm{HbAlc}$ ( $\geq$ the average value $9.61 \%$ ), hypertension, smoking, reduced blood HDL-c levels $(<1.04 \mathrm{mmol} / \mathrm{L}$ (men) or $<1.29 \mathrm{mmol} / \mathrm{L}$ (women)). The independent variables were as follows: UKPDS CHD risk ( $>20 \%$, high risk, $1 ; \leq 20 \%, 0)$ and stroke risk (>10\%, high risk, $1 ; \leq 10 \%, 0)$. The results showed that PAD was an independent risk factor for $\mathrm{CHD}$ (odds ratio: 3.6, 95\% CI: 2.2-6.0, $P=0.000$ ) and stroke (odds ratio: 6.9, 95\% CI: 4.0-11.8, $P=0.000$; Table 4).

\section{Discussion}

The ABI is a simple, inexpensive, and noninvasive method of detecting lower extremity PAD in diabetes patients. Various ABI cutoffs have been proposed for detecting PAD in different studies. The 2011 ACCF/AHA guidelines set the ABI cutoff at $\leq 0.9$; in addition, they stated that an $\mathrm{ABI}>1.3$ suggested atherosclerosis, while an $\mathrm{ABI}>1.4$ indicated cardiovascular risk $[3,20,21]$. In this study, $81(6.9 \%)$ patients 

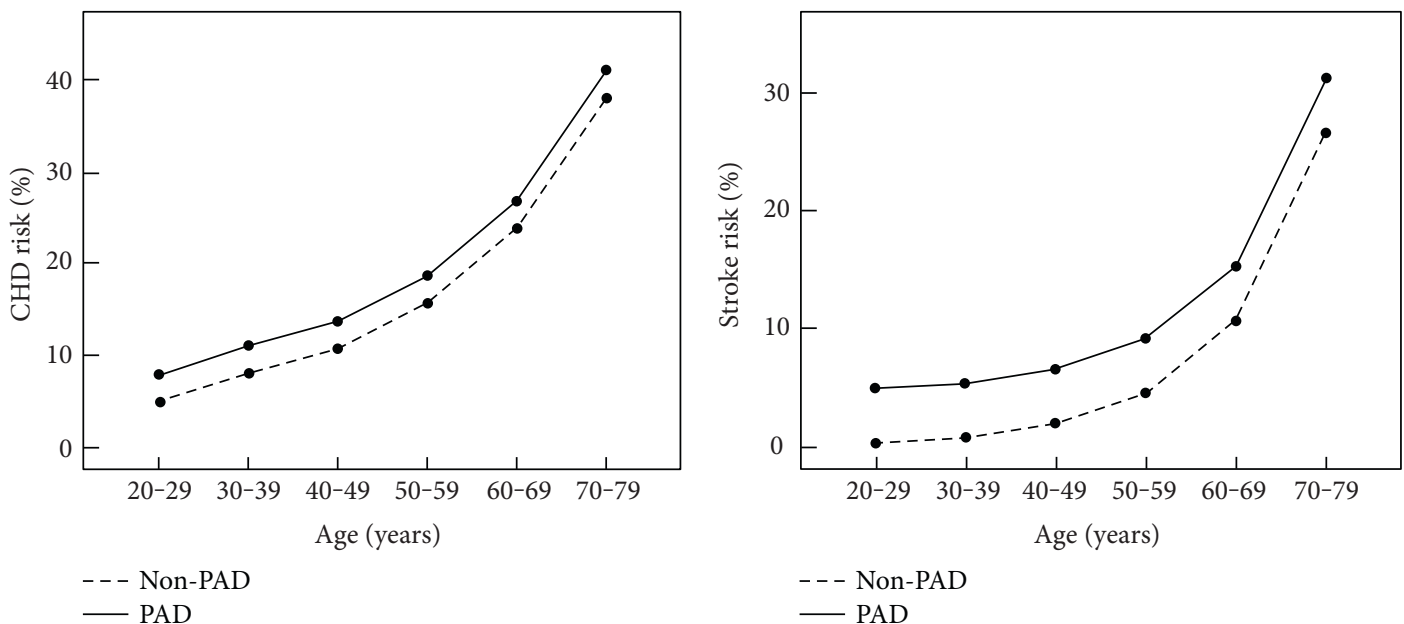

FIGURE 1: Age-related prevalence of CHD risk and stroke risk in diabetes patients. CHD: coronary heart disease; PAD: peripheral arterial disease; $\mathrm{ABI}$ : ankle-brachial index. $\mathrm{PAD}$ group: $\mathrm{ABI} \leq 0.9$ or $\mathrm{ABI}>1.4$; non-PAD group: $0.9<\mathrm{ABI} \leq 1.4$.

TABLE 3: Multivariate linear regression analysis of risk factors for CHD and stroke as estimated using the UKPDS risk engine.

\begin{tabular}{|c|c|c|c|c|c|c|c|c|}
\hline \multirow[t]{2}{*}{ Variables } & \multicolumn{2}{|c|}{ UKPDS CVD risk } & \multicolumn{2}{|c|}{ UKPDS stroke risk } & \multicolumn{2}{|c|}{$\begin{array}{l}\text { UKPDS fatal } \\
\text { CVD risk }\end{array}$} & \multicolumn{2}{|c|}{$\begin{array}{l}\text { UKPDS fatal } \\
\text { stroke risk }\end{array}$} \\
\hline & Beta & $P$ & Beta & $P$ & Beta & $P$ & Beta & $P$ \\
\hline Male & -0.355 & 0.000 & -0.168 & 0.000 & -0.304 & 0.000 & -0.142 & 0.000 \\
\hline Age & 0.726 & 0.000 & 0.602 & 0.000 & 0.711 & 0.000 & 0.507 & 0.000 \\
\hline BMI & -0.023 & 0.040 & 0.004 & NS & -0.023 & NS & -0.004 & NS \\
\hline Duration & 0.081 & 0.000 & 0.270 & 0.000 & 0.148 & 0.000 & 0.248 & 0.000 \\
\hline SBP & 0.082 & 0.000 & 0.044 & NS & 0.101 & 0.000 & 0.232 & 0.000 \\
\hline DBP & -0.020 & NS & 0.000 & NS & -0.025 & NS & -0.022 & NS \\
\hline HbAlc & 0.352 & 0.000 & 0.031 & NS & 0.363 & 0.000 & 0.041 & 0.025 \\
\hline $\mathrm{LDL}-\mathrm{c}$ & -0.024 & NS & -0.022 & NS & -0.016 & NS & -0.022 & NS \\
\hline $\mathrm{HDL}-\mathrm{c}$ & -0.285 & 0.000 & -0.045 & 0.050 & -0.233 & 0.000 & -0.043 & NS \\
\hline TC & 0.302 & 0.000 & 0.061 & NS & 0.237 & 0.000 & 0.050 & NS \\
\hline TG & 0.000 & NS & 0.032 & NS & -0.004 & NS & 0.024 & NS \\
\hline Smoking & 0.051 & 0.000 & 0.019 & NS & 0.014 & NS & 0.018 & NS \\
\hline PAD & 0.055 & 0.000 & 0.140 & 0.000 & 0.066 & 0.019 & 0.140 & 0.000 \\
\hline
\end{tabular}

NS: not significant; BMI: body mass index; SBP: systolic blood pressure; DBP: diastolic blood pressure; HDL-c: high-density lipoprotein cholesterol; LDL-c: lowdensity lipoprotein cholesterol; PAD: peripheral arterial disease; ABI: ankle-brachial index; TG: triglyceride; TC: total cholesterol; HbA1c: glycosylated hemoglobin; UKPDS: United Kingdom Prospective Diabetes Study; CHD: coronary heart disease.

TABLE 4: Multivariate binary logistic regression analysis of risk factors for CHD and stroke as estimated using the UKPDS risk engine.

\begin{tabular}{|c|c|c|c|c|}
\hline \multirow{2}{*}{ Variables } & \multicolumn{2}{|c|}{$\mathrm{CHD}$ risk } & \multicolumn{2}{|c|}{ Stroke risk } \\
\hline & OR (95\% CL) & $P$ & OR (95\% CL) & $P$ \\
\hline Age $\geq 50$ years & $33.2(20.2-54.4)$ & 0.000 & $255.1(35.5-1832.4 .5)$ & 0.000 \\
\hline Hypertension & $2.0(1.5-2.8)$ & 0.000 & $2.1(1.5-2.8)$ & 0.000 \\
\hline Smoking & $5.5(3.9-7.6)$ & 0.000 & $1.5(1.1-2.0)$ & 0.013 \\
\hline Elevated $\mathrm{HbA} 1 \mathrm{c}$ & $4.5(3.4-6.1)$ & 0.000 & $0.8(0.6-1.1)$ & 0.182 \\
\hline Reduced HDL-c & $1.4(1.1-1.9)$ & 0.012 & $0.9(0.7-1.2)$ & 0.594 \\
\hline $\mathrm{PAD}$ & $3.6(2.2-6.0)$ & 0.000 & $6.9(4.0-11.8)$ & 0.000 \\
\hline
\end{tabular}

UKPDS: United Kingdom Prospective Diabetes Study; CHD: coronary heart disease. Elevated HbAlc ( $\geq$ the average value 9.61\%); reduced HDL-c $(<1.04 \mathrm{mmol} / \mathrm{L}$ (men) or $<1.29 \mathrm{mmol} / \mathrm{L}$ (women)). 
had an $\mathrm{ABI} \leq 0.9$ and 7 (0.6\%) patients had an $\mathrm{ABI}>1.4$; the rate of lower extremity PAD is lower than the rates reported previously [19]. This result could be due to inclusion of young diabetic patients and exclusion of the population with $\mathrm{CHD}$ and cerebrovascular disease in our study. The value of the ABI is related to age. According to Fowkes et al., in developing countries, the incidence of a low ABI among 45-49 year olds was $6.31 \%$ in women and $2.89 \%$ in men; in contrast, the incidence among $85-89$ year olds was $15.22 \%$ in women and $14.94 \%$ in men [19].

This study showed that the proportion of women with a low ABI was significantly higher than that of men with a low ABI, which is consistent with previous literature [22, 23]. The San Luis Valley Diabetes Study showed that in the absence of traditional risk factors for cerebrovascular disease, the average $\mathrm{ABI}$ was 0.07 points lower in women than in men [22]. In the Multi-Ethnic Study of Atherosclerosis (MESA), which included patients without PAD and traditional atherosclerosis risk factors, the $\mathrm{ABI}$ was 0.02 points lower in women than in men after adjustment for multiple variables [23].

Our study also demonstrated that the 10-year CHD and stroke risks were significantly greater in the PAD group than in the non-PAD group, and the $\mathrm{ABI}$ was an independent predictor of the 10-year CHD and stroke risks. In addition to the diagnosis of $\mathrm{PAD}$, the $\mathrm{ABI}$ is associated with cardiovascular risk factors and cardiovascular events. A low ABI has been related to many known cardiovascular risk factors, including hypertension, diabetes, smoking, dyslipidemia, obesity, and C-reactive protein [5-7]. Several population-based cohort studies have confirmed that a decrease in an ABI is highly correlated with the prevalence rate of coronary artery disease and cerebrovascular disease [8-11], which indicates that the $\mathrm{ABI}$ is an independent risk factor for cardiovascular and cerebrovascular disease. A few studies have indicated that an $\mathrm{ABI}>1.40$ is associated with stroke and CHD. The curve obtained by plotting ABI values on the $x$-axis and mortality and other cardiovascular events on the $y$-axis appears as a reverse J curve, in which the risk is lowest in the ABI range of 1.11-1.40 [3, 16, 24]. Reports have indicated that an abnormal $A B I$ in patients with diabetes or metabolic syndrome is related to cardiovascular events and risk factors $[1,9,16$, 17]. A study from Hong Kong found that in diabetes patients, ABI values of 0.91-0.99 were associated with increased microvascular and macrovascular complications [18].

Considering that age is the most important factor affecting the $\mathrm{ABI}$ and $\mathrm{CHD}$ and stroke risks, we stratified the patients by age and recalculated the CHD and stroke risks. The results showed that the cardiovascular risk was higher in the PAD group than in the non-PAD group for every age group, which indicated that an abnormal ABI predicts $\mathrm{CHD}$ and stroke risks independent of age.

Furthermore, the combination of the ABI in cardiovascular risk stratification with the current methods for predicting the 10-year risk of cardiovascular disease would improve risk prediction. The ABI Collaboration conducted a metaanalysis of 16 cohort studies based on individuals, focusing on whether the $\mathrm{ABI}$ can predict the risk of cardiovascular events and death independently from the FRS and whether it can improve risk prediction when used in combination with the FRS [25]. The results showed that the use of the ABI would lead to a reclassification of the risk levels for men and women. This is consistent with the findings of the MESA study. The FRS is mainly used for the general population, while the UKPDS risk score is used for patients with diabetes [2]. There have been many studies on the correlation between the ABI and FRS in diabetes patients [26], but relatively few on the ABI and UKPDS risk score in diabetes patients. The ABI combining with the UKPDS risk engine for prediction of $\mathrm{CHD}$ and stroke risks in diabetes patients needs a further study.

This study has some limitations. First, the study involved only hospitalized patients, many of whom had poor glycemic control. However, at present, glycemic control is less than ideal all over the world. A cross-sectional study of 9065 T2DM outpatients from 26 medical centers in China found that blood glucose levels were controlled in only $32.6 \%$ of patients [27],which was similar to the rate of $31.78 \%$ among 238,639 diabetes patients reported by Ji et al. in 2013 [28]. The International Diabetes Mellitus Practice Study [29] included 11,799 patients from 17 countries in Eastern Europe, Africa, South America, and Latin America and found a blood glucose-control rate of only $25 \%$. Thus, the glycemic control in our patients may reflect that observed in most diabetes patients. Second, the UKPDS risk engine originated from British diabetes patients, and whether or not it is suitable for Chinese patients remains to be investigated. However, it is currently an established risk assessment tool worldwide.

In conclusion, our study found that the 10 -year $\mathrm{CHD}$ and stroke risks were higher in diabetes patients with lower extremity PAD than in diabetes patients without PAD, and lower extremity PAD was an independent risk factor for cardiovascular diseases in diabetes patients. Given that the ABI is a simple and easy method of detecting lower extremity $\mathrm{PAD}, \mathrm{ABI}$ measurements will be beneficial for the estimation of cardiovascular disease and stroke risks in T2DM patients.

\section{Conflicts of Interest}

The authors declare that there is no conflict of interests regarding the publication of this paper.

\section{Authors' Contributions}

Xiao-Hong Pang and Jue Han contributed equally.

\section{Acknowledgments}

This study was supported in part by funding from the Chinese Society of Endocrinology (13040620447), the National Natural Science Foundation of China (81370968, 81670744), and the Foundation of Education Department of Zhejiang Province of China (Y201328533).

\section{References}

[1] P. E. Norman, W. A. Davis, D. G. Bruce, and T. M. Davis, "Peripheral arterial disease and risk of cardiac death in type 2 diabetes: the Fremantle Diabetes Study," Diabetes Care, vol. 29, no. 3, pp. 575-580, 2006. 
[2] D. Mukherjee, "Peripheral and cerebrovascular atherosclerotic disease in diabetes mellitus," Best Practice \& Research Clinical Endocrinology \& Metabolism, vol. 23, no. 3, pp. 335-345, 2009.

[3] V. Aboyans, M. H. Criqui, P. Abraham et al., "Measurement and interpretation of the ankle-brachial index: a scientific statement from the American Heart Association," Circulation, vol. 126, no. 24, pp. 2890-2909, 2012.

[4] M. Rac-Albu, L. Iliuta, S. M. Guberna, and C. Sinescu, “The role of ankle-brachial index for predicting peripheral arterial disease," Maedica, vol. 9, no. 3, pp. 295-302, 2014.

[5] K. Sozmen and B. Unal, "Prevalence of low ankle brachial index and relationship with cardiovascular risk factors in a Western urban population in Turkey," Angiology, vol. 65, no. 1, pp. 43-50, 2014.

[6] P. F. de Albuquerque, P. H. O. de Albuquerque, G. O. de Albuquerque, D. M. Servantes, S. M. Carvalho, and J. A. Oliveira Filho, "Ankle-brachial index and ventricular hypertrophy in arterial hypertension," Arquivos Brasileiros de Cardiologia, vol. 98, no. 1, pp. 84-86, 2012.

[7] G. H. Tison, C. E. Ndumele, G. Gerstenblith, M. A. Allison, J. F. Polak, and M. Szklo, "Usefulness of baseline obesity to predict development of a high ankle brachial index (from the Multi-Ethnic Study of Atherosclerosis)," The American Journal of Cardiology, vol. 107, no. 9, pp. 1386-1391, 2011.

[8] J. B. Hong, C. O. Leonards, M. Endres, B. Siegerink, and T. G. Liman, "Ankle-brachial index and recurrent stroke risk: metaanalysis," Stroke, vol. 47, no. 2, pp. 317-322, 2016.

[9] Y. Cang, J. Li, Y. M. Li et al., "Relationship of a low anklebrachial index with all-cause mortality and cardiovascular mortality in Chinese patients with metabolic syndrome after a 6-year follow-up: a Chinese prospective cohort study," Internal Medicine, vol. 51, no. 20, pp. 2847-2856, 2012.

[10] B. Ovbiagele, "Association of ankle-brachial index level with stroke," Journal of the Neurological Sciences, vol. 276, no. 1-2, pp. 14-17, 2009.

[11] S. Sen, D. R. Lynch, E. Kaltsas et al., "Association of asymptomatic peripheral arterial disease with vascular events in patients with stroke or transient ischemic attack," Stroke, vol. 40, no. 11, pp. 3472-3477, 2009.

[12] P. W. Wilson, R. B. D'Agostino, D. Levy, A. M. Belanger, H. Silbershatz, and W. B. Kannel, "Prediction of coronary heart disease using risk factor categories," Circulation, vol. 97, no. 18, pp. 1837-1847, 1998.

[13] R. L. Coleman, R. J. Stevens, R. Retnakaran, and R. R. Holman, "Framingham, SCORE, and DECODE risk equations do not provide reliable cardiovascular risk estimates in type 2 diabetes," Diabetes Care, vol. 30, no. 5, pp. 1292-1293, 2007.

[14] D. C. Goff, D. M. Lloyd-Jones, G. Bennett et al., "2013 ACC/ AHA guideline on the assessment of cardiovascular risk: a report of the American College of Cardiology/American Heart Association Task Force on practice guidelines," Journal of the American College of Cardiology, vol. 63, no. 25, pp. 2935-2959, 2014.

[15] R. J. Stevens, V. Kothari, A. I. Adler, I. M. Stratton, and United Kingdom Prospective Diabetes Study (UKPDS) Group, "The UKPDS risk engine: a model for the risk of coronary heart disease in type II diabetes (UKPDS 56)," Clinical Science, vol. 101, no. 6, pp. 671-679, 2001.

[16] Q. Li, H. Zeng, F. Liu et al., "High ankle-brachial index indicates cardiovascular and peripheral arterial disease in patients with type 2 diabetes," Angiology, vol. 66, no. 10, pp. 918-924, 2015.
[17] N. M. J. Hanssen, M. S. Huijberts, C. G. Schalkwijk, G. Nijpels, J. M. Dekker, and C. D. Stehouwer, "Associations between the ankle-brachial index and cardiovascular and all-cause mortality are similar in individuals without and with type 2 diabetes nineteen-year follow-up of a population-based cohort study," Diabetes Care, vol. 35, no. 8, pp. 1731-1735, 2012.

[18] B. P. Yan, Y. Y. Zhang, A. P. S. Kong et al., "Borderline anklebrachial index is associated with increased prevalence of micro- and macrovascular complications in type 2 diabetes: a cross-sectional analysis of 12,772 patients from the Joint Asia Diabetes Evaluation Program," Diabetes and Vascular Disease Research, vol. 12, no. 5, pp. 334-341, 2015.

[19] F. G. Fowkes, D. Rudan, I. Rudan et al., "Comparison of global estimates of prevalence and risk factors for peripheral artery disease in 2000 and 2010: a systematic review and analysis," Lancet, vol. 382, no. 9901, pp. 1329-1340, 2013.

[20] F. G. Fowkes, "The measurement of atherosclerotic peripheral arterial disease in epidemiological surveys," International Journal of Epidemiology, vol. 17, no. 2, pp. 248-254, 1988.

[21] Writing Group M, Writing Committee M, and Accf/Aha Task Force M, "2011 ACCF/AHA focused update of the guideline for the management of patients with peripheral artery disease (updating the 2005 guideline): a report of the American College of Cardiology Foundation/American Heart Association Task Force on practice guidelines," Circulation, vol. 124, no. 18, pp. 2020-2045, 2011.

[22] W. R. Hiatt, S. Hoag, and R. F. Hamman, "Effect of diagnostic criteria on the prevalence of peripheral arterial disease. The San Luis Valley Diabetes Study," Circulation, vol. 91, no. 5, pp. 1472-1479, 1995.

[23] V. Aboyans, M. H. Criqui, R. L. McClelland et al., "Intrinsic contribution of gender and ethnicity to normal anklebrachial index values: the Multi-Ethnic Study of Atherosclerosis (MESA)," Journal of Vascular Surgery, vol. 45, no. 2, pp. 319-327, 2007.

[24] E. J. E. Hendriks, J. Westerink, P. A. de Jong et al., “Association of high ankle brachial index with incident cardiovascular disease and mortality in a high-risk population," Arteriosclerosis, Thrombosis, and Vascular Biology, vol. 36, no. 2, pp. 412-417, 2016.

[25] Ankle Brachial Index Collaboration, F. G. Fowkes, G. D. Murray et al.I. Butcher, C. L. Heald, R. J. Lee et al., "Ankle brachial index combined with Framingham Risk Score to predict cardiovascular events and mortality: a meta-analysis," Jama, vol. 300, no. 2, pp. 197-208, 2008.

[26] M. S. Amer, M. S. Khater, O. H. Omar, R. A. Mabrouk, and W. H. El-Kawaly, "Framingham risk score and ankle-brachial index in diabetic older adults," International Journal of Cardiology, vol. 168, no. 2, pp. 1620-1621, 2013.

[27] R. Chen, L. Ji, L. Chen et al., "Glycemic control rate of T2DM outpatients in China: a multi-center survey," Medical Science Monitor, vol. 21, pp. 1440-1446, 2015.

[28] L. N. Ji, J. M. Lu, X. H. Guo et al., "Glycemic control among patients in China with type 2 diabetes mellitus receiving oral drugs or injectables," BMC Public Health, vol. 13, no. 1, p. $602,2013$.

[29] J. C. Chan, J. J. Gagliardino, S. H. Baik et al., "Multifaceted determinants for achieving glycemic control: the International Diabetes Management Practice Study (IDMPS)," Diabetes Care, vol. 32, no. 2, pp. 227-233, 2009. 


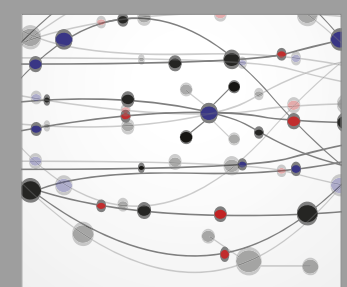

The Scientific World Journal
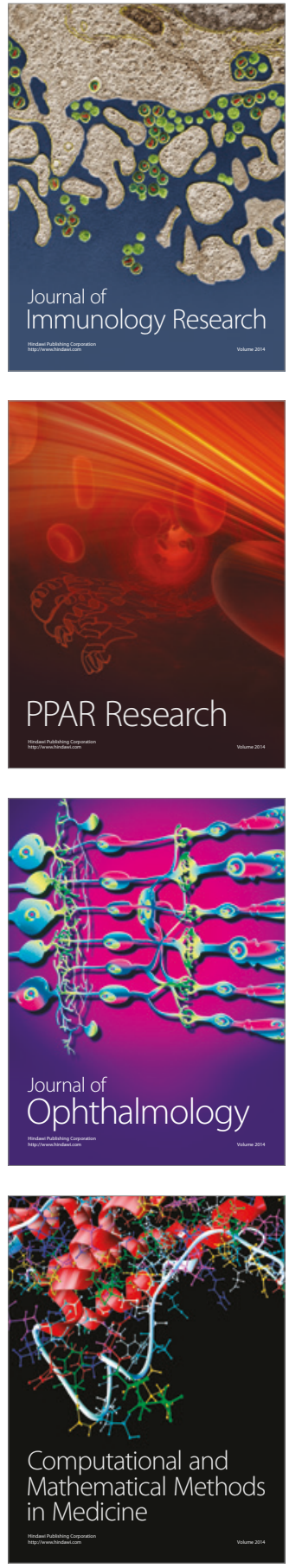

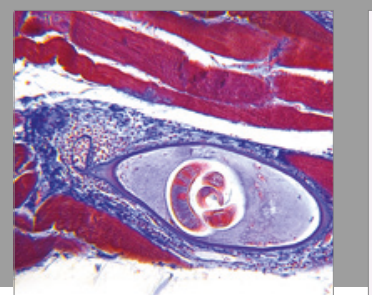

Gastroenterology Research and Practice
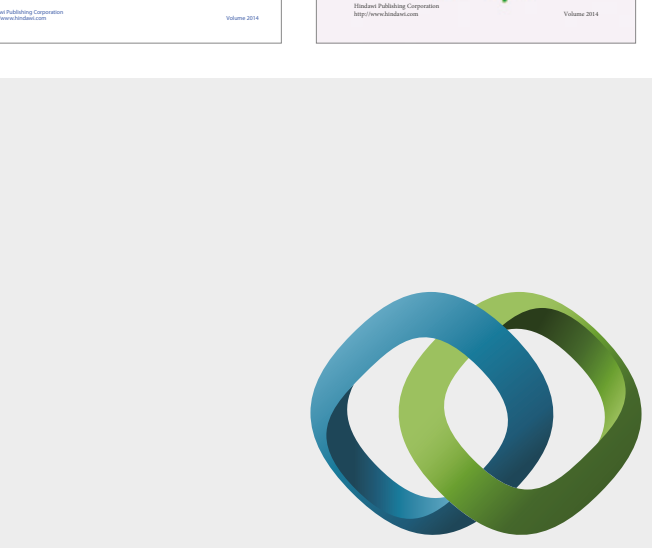

\section{Hindawi}

Submit your manuscripts at

https://www.hindawi.com
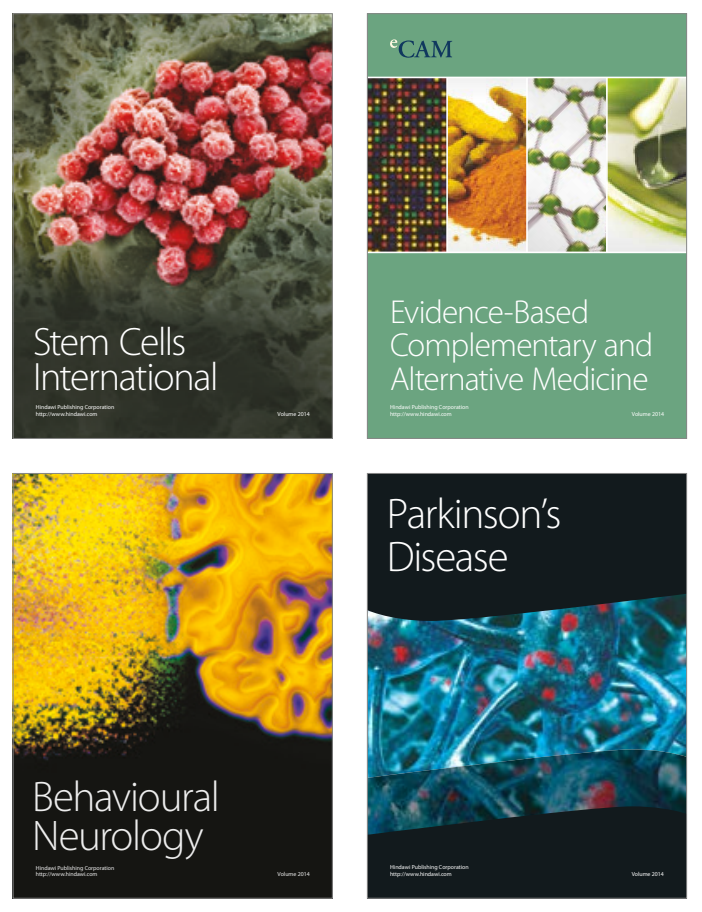
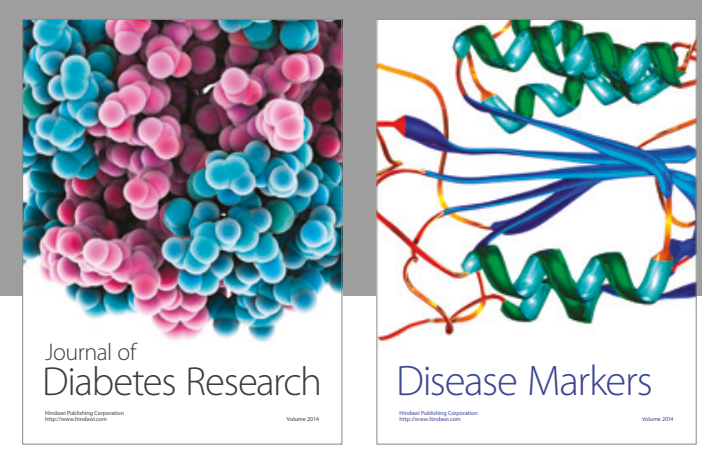

Disease Markers
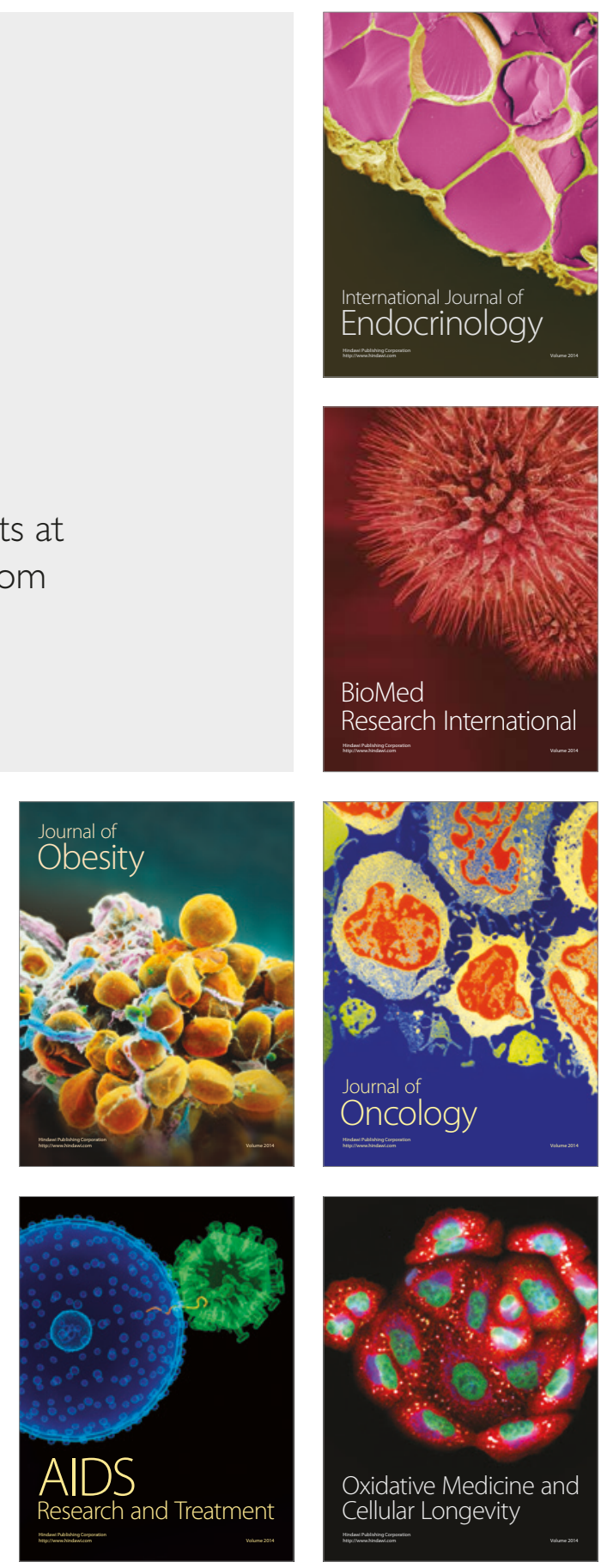